ARTICLE

Check for updates

\title{
Low-grade oncocytic renal tumor (LOT): mutations in mTOR pathway genes and low expression of FOXI1
}

\author{
Aurélien Morini ${ }^{1,2}$, Tom Drossart ${ }^{2}$, Marc-Olivier Timsit ${ }^{2,3}$, Mathilde Sibony ${ }^{4}$, Viorel Vasiliu ${ }^{5}$, Anne-Paule Gimenez-Roqueplo (iD ${ }^{2,6,7}$, \\ Judith Favier $\mathbb{D}^{2}$, Cécile Badoual ${ }^{1}$, Arnaud Mejean ${ }^{3,7}$, Nelly Burnichon ${ }^{2,6,8}$ and Virginie Verkarre (iD) ${ }^{1,2,7,8}$ 的
}

(c) The Author(s), under exclusive licence to United States \& Canadian Academy of Pathology 2021

Low-grade oncocytic renal tumor (LOT) is an emerging provisional entity, described as rare solid renal oncocytic/eosinophilic tumor sharing diffuse CK7 and negative CD117 immunoprofile. The links between LOT and other eosinophilic chromophobe like-renal cell carcinomas (RCC) are currently discussed. We sequenced tumoral DNA with a next generation sequencing panel for kidney cancer and carried out immunohistochemical analyses with CK7, CD117, SDHB, 4EBP1-P, S6K-P, and FOXI1 antibodies in a series of ten cases of LOT (9 females, 1 male; mean age at surgery: 66 years, 42.3 to 83.4) retrospectively diagnosed from a cohort of 272 tumors initially classified as chromophobe RCC (CHRCC). All LOT were single, without known hereditary predisposition, classified stage pT1 (70\%), pT2 (20\%) or pT3a (10\%). Morphological features were similar to previous descriptions and clinical behavior was indolent for the six cases with available follow-up. We identified genetic variations in mTOR pathway related genes in $80 \%$ of cases, MTOR $(7$ cases) or TSC1 (1 case). Expression of FOXI1 was absent in all cases. In 9 LOT, 4EBP1-P and S6K-P were overexpressed, suggesting mTOR pathway activation.

Our data highlights the major role of mTOR pathway in tumorigenesis of LOT mostly due to activating MTOR gene variations. Absence of FOXI1 expression is a strong argument to distinguish LOT from eosinophilic CHRCC and to bring them closer to other recently described FOXI1 negative eosinophilic-CHRCC like with MTOR/TSC mutations. Altogether, our data argue to consider LOT as a distinct entity with a favorable clinical outcome. However, in case of metastasis, an accurate diagnosis of LOT would be essential for the patient's management and could allow targeted therapy.

Modern Pathology (2022) 35:352-360; https://doi.org/10.1038/s41379-021-00906-7

\section{INTRODUCTION}

Low-grade oncocytic renal tumor (LOT) is rare oncocytic/ eosinophilic renal tumor firstly described in 2019 in a cohort of 28 patients $^{1}$ and recently proposed to be considered as a provisional entity by the Genitourinary Pathology Society (GUPS) ${ }^{2}$. With a total of 93 cases published until now in three additional cohorts from 8 to 29 patients, LOT has been described mainly as isolated tumor with sporadic presentation occurring at mean age of 56 to 67 years (range $39-84)(44 M / 49 F)^{1,3-6}$. All tumors had indolent clinical behavior ${ }^{1,6}$. Recently, four cases of multiple LOT occurring in Tuberous Sclerosis complex (TSC) setting have been reported for the first time $e^{4,5}$. LOT is solid renal tumor sharing homogeneous diffuse positive CK7 and negative CD117 immunoprofile that distinguish it from most of the chromophobe renal cell carcinoma (CHRCC) and from renal oncocytoma (RO). Nuclear features are close to those of RO with low-grade nuclei, and lack irregularities classically observed in $\mathrm{CHRCC}^{1}$. Oncocytic/eosinophilic cytoplasms of LOT can contain focal perinuclear halos without vacuoles. Their accurate diagnosis is challenging among the spectrum of eosinophilic renal tumors with "oncocytic" features but is essential as it may lead to better prognosis or more appropriate clinical management ${ }^{2}$. Considering that diffuse CK7 positivity is shared by LOT and most $\mathrm{CHRCC}^{7-12}$ and allows the exclusion of RO diagnosis (negative CK7 or restricted to scattered individual cells) ${ }^{9}$, the pure eosinophilic variant of CHRCC may represent the main differential diagnosis of LOT. CD117, a lineagespecific marker expressed by intercalated cells in the distal nephron and shared by most RO and $\mathrm{CHRCC}^{7,8,10,11,13}$ is negative in LOT. As sensitivity of CD117 in CHRCC remains limited to $90 \%{ }^{10,14-16}$, other diagnostic biomarkers could be useful to distinguish LOT from CHRCC and might contribute to classify LOT as a distinct entity. Among them, the transcription factor FOXI1 (Forkhead box 11) which represents a newly identified biomarker for intercalated cells of distal nephron ${ }^{17}$ is, like CD117, specifically expressed in both RO and $\mathrm{CHRCC}^{17-19}$ but was not previously evaluated in LOT.

\footnotetext{
${ }^{1}$ Hôpital européen Georges Pompidou, Université de Paris, F-75006, Paris, Department of Pathology, F-75015 Paris, France. ${ }^{2}$ Université de Paris, PARCC, INSERM, Equipe Labellisée

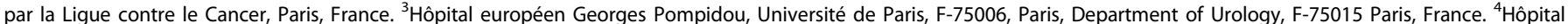

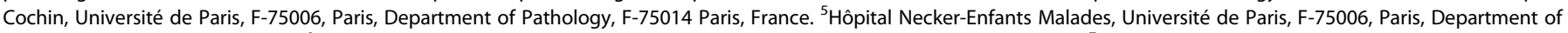

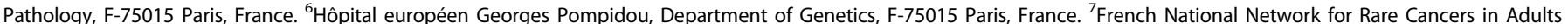

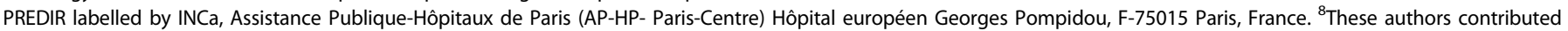

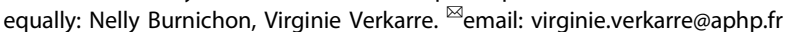

Received: 25 May 2021 Revised: 13 August 2021 Accepted: 13 August 2021

Published online: 16 September 2021 
Interestingly, link between LOT and a subset of recently described eosinophilic chromophobe like-RCC has been suggested in two recent GUPS reviews ${ }^{2,20}$. Eosinophilic variant of CHRCC represents $20 \%$ to $25 \%$ of CHRCC series ${ }^{21}$ and are composed of smaller eosinophilic cells harboring less uncommonly round regular nuclei ${ }^{22,23}$. Negative or low FOXI1 mRNA and protein expression have been recently found in four eosinophilic tumors from the TCGA CHRCC cohort $(\mathrm{KICH})$ as well as in two newly identified tumors mimicking eosinophilic CHRCC $^{18,19}$. Their morphological features seem close to those of LOT. These tumors also harbor genetic alterations in MTOR or $T S C 1 / 2$ genes that differ from main genetic features involved in $\mathrm{CHRCC}^{24}$. Besides, their copy number chromosomal pattern is mostly disomic, as up to $41 \%$ of eosinophilic CHRCC and contrast with the classic pattern of multiple entire chromosomal losses in $\mathrm{CHRCC}^{21,25}$. Profile of copy number alterations analyzed in 9 cases of LOT differed from classic pattern of CHRCC and presented either partial losses including recurrent deletions of $19 p 13.3(7 / 9), 1$ p36.33 (5/9), and 19q13.11 (4/9) or a disomic pattern $(2 / 9)^{1}$.

Finally, except for one case of MTOR somatic mutation we briefly reported recently in $\mathrm{LOT}^{26}$, mutational analysis has not been yet investigated in LOT.

With the objective to evaluate whether the mTOR pathway could play a driver role in the tumorigenesis of LOT, we performed a genetic study in 10 cases of these rare tumors. We also carried out an extensive clinicopathological and immunohistochemical characterization as well as the expression study of FOXI1 protein, with the aim to clarify the hypothesis that LOT may represent a distinct entity from eosinophilic CHRCC.

\section{MATERIALS AND METHODS}

Our study was approved by the French national ethics committee (Comité de Protection des Personnes Number 19.05.27.61541; national identification 2018-A03147-48).

\section{Selection of cases}

Ten cases of LOT were identified retrospectively from a series of 272 surgical archival cases previously diagnosed as CHRCC from Necker Enfants-Malades Hospital and European Georges Pompidou Hospital (APHP Centre-Université de Paris) embedded in 13 tissue micro-array (TMA) of formalin fixed paraffin embedded (FFPE) tissue blocks (data not shown). One core with a diameter of $2.0 \mathrm{~mm}$ was punched out from two blocks/tumor. As initially described by Trpkov et al. ${ }^{1}$, diagnosis of LOT was established for cases combining diffuse positive CK7 and negative CD117 staining and morphological criteria as follows: eosinophilic/ oncocytic cells, solid architecture, low grade nucleoli, few perinuclear halos without significant irregular/raisinoid nuclei. After the selection of cases based on TMA results, the final diagnosis of LOT was confirmed on whole slides for each case.

\section{Clinical, pathological, and immunohistochemical data}

Clinical data of the 10 patients affected by LOT were collected using the medical records including age, gender, tumor size, stage, number of tumors, surgical procedure, follow-up, associated renal tumor or cyst, and personal or familial history evocating a hereditary form of the disease. Pathological features were analyzed by reviewing all hematoxylin, eosin, saffron (HES) on whole slide by two senior uropathologists (VV-MS) and one junior pathologist (AM).

Immunohistochemistry was performed on Ventana BenchMark Ultra autostainer (Ventana Medical Systems) using the following primary antibodies: CK7 antibody (Dako, M7018, 1/50), CD117 antibody (Dako, A4505, 1/100), SDHB antibody (Sigma, HPA002868, 1/250), FOXI1 (OriGene Technologies, Rockville, MD, USA, clone OTI2G6, 1/50), and two antibodies downstream the mTOR pathway: Phospho-S6 Ribosomal Protein (Ser240/244) antibody (Cell Signaling, D68F8, 1/300) and Phospho-4EBP1 (Thr37/46) antibody (Cell Signaling, 236B4, 1/100). Immunohistochemistry was performed on 13 FFPE TMA blocks with 4- $\mu \mathrm{m}$-thick tissue sections for all antibodies (data not shown for others 262 CHRCC included in the cohort).
Expression of FOXI1 was graded as performed in Tong et al. study according to the percentage of tumor cells with nuclear staining (negative $<1 \%, 1 \% \leq$ focal $\leq 25 \%, 26 \% \leq$ moderate $\leq 50 \%$, diffuse $>50 \%$ ). The intensity of nuclear staining was also recorded as 'weak', 'intermediate' or 'strong'19. Nuclear staining on intercalated cells of distal nephron was used as positive internal control in peritumoral kidney when available or in corresponding TMA. Expression of S6K-P, 4EBP1-P was evaluated on TMA with others CHRCC (data not shown for CHRCC) by an H-score which combined intensity $\times$ percentage of positive cells for each intensity score. $\mathrm{H}$-score was considered as negative or low when $<100 / 300$ and significantly positive and overexpressed when $\geq 100 / 300$ ( + if $\geq 100 / 300$ and $<200 / 300$ and ++ if $\geq 200 / 300$ ).

\section{Genetic analysis}

Ten LOT were analyzed for somatic variations in genes involved in kidney tumor predisposition or in renal tumorigenesis. Tumor DNA extraction was performed using an AllPrep DNA/RNA Mini kit (Qiagen $\left.{ }^{\circ}\right)$ for frozen tumors $(n=2)$ or using a Maxwell 16 Instrument (Promega) with Maxwell 16 FFPE Plus LEV DNA Purification Kit (Promega) for FFPE tumor tissue $(n=8)$. In addition, two patients (\#1 and \#2) benefited from a germline DNA analysis after genetic counseling and signature of informed consent.

Somatic and germline genetic analyses were performed by next-generation sequencing (NGS) using an in-house panel of 26 genes (SeqCap EZ HyperCap, Roche ${ }^{\circ}$ ) comprising BAP1, CDKN2A, CDKN2B, FH, FLCN, HNF1B, KDM5C, KDM6A, MET, MITF, MTOR, NF2, PBRM1, PTEN, SDHA, SDHB, SDHC, SDHD, SETD2, SMARCB1, TCEB1, TMEM127, TP53, TSC1, TSC2, and VHL ${ }^{24}$. DNA libraries were prepared according to the manufacturer's recommendations and $2 \times 150 \mathrm{bp}$ paired end sequenced on MiSeq platform (Illumina, CA, USA) using v2 chemistry according to the standard protocol. Demultiplexing was performed using MiSeq Reporter (Illumina, CA, USA). Alignment to the human genome hg19 (GRch37) and variant calling was performed using SeqNext (JSI Medical Systems) and PolyDiag (Paris Descartes University, France) software.

Variants of interest found by NGS were confirmed by Sanger sequencing. Variant annotation was mainly performed using Alamut Visual 2.7 (Interactive Biosoftware) as an interface. To assess the frequency and the impact of variants of interest identified by NGS, the following databases were consulted: Cancer Genome Interpreter (https://www. cancergenomeinterpreter.org/mutations), ClinVar (https://www-ncbi-nlmnih-gov.proxy.insermbiblio.inist.fr/clinvar/), COSMIC (https://cancer.sanger. ac.uk/cosmic/gene/analysis?ln=MTOR), OncoKB (https://www.oncokb.org/ ), genome aggregation database gnomAD (https://gnomad.broadinstitute. org/gene/ENSG00000105976?dataset=gnomad r2 1), and TSC1 Leiden Open Variation Database (LOVD) (https://databases.lovd.nl/shared/genes/ TSC1). Variants were considered as 'pathogenic' when they had been reported as causal mutations with evidence, or 'variant of uncertain significance' (VUS) when they had never been reported.

\section{RESULTS}

\section{Clinical presentation}

The cohort included 10 patients with mean age at diagnosis of 66 years (42-83) and female predominance (M:F ratio = 1:9) (Table 1). No familial history of renal tumors was noticed nor hereditary setting.

All patients had single renal tumor ( 4 on left and 6 on right kidney) resected by radical (3/10) or partial nephrectomy (7/10). Follow-up was available in 6 out of 10 patients with a mean of 46 months (7-90) without evidence of disease recurrence, lymph node invasion or metastasis. One patient died of cardiovascular disease (Table 1).

\section{Pathological features}

On gross examination, the tumor cut was beige/mahogany and solid (Supplementary Fig. 1). Tumors had an average size of $4.8 \mathrm{~cm}(2.5-8.5)$ classified as pT1a or pT1b $(80 \%, 8 / 10)$ (Table 1). Main microscopic features were summarized on Supplementary Table 1. One tumor was pT2a and one pT3a due to focal sinus involvement without vascular extension (Fig. 1A). All tumors were well-circumscribed (Fig. 1B). Only 30\% (3/10) were partially encapsulated (Fig. 1C). All tumors were made up of compact small nests (about 10 to 30 cells by nest) associated with trabecular architecture $(40 \%, 4 / 10)$ (Fig. 1D) or focal microcysts 
Table 1. Clinicopathological data of LOTs.

\begin{tabular}{|c|c|c|c|c|c|c|c|c|c|}
\hline Case & Age (years) & Gender & $\mathbf{N}+/ \mathbf{M}+$ & Nephrectomy & Side & Tumor size $(\mathrm{cm})$ & Stade & Follow up (months) & Status \\
\hline 1 & 57 & $\mathrm{~F}$ & $-/-$ & Partial & Left & 3.7 & pT1a & 7 & AWD \\
\hline 2 & 61 & $\mathrm{~F}$ & $-/$ & Partial & Right & 3.8 & pT1a & NA & NA \\
\hline 3 & 56 & $\mathrm{~F}$ & $-/-$ & Partial & Left & 2.5 & pT1a & 43 & AWD \\
\hline 4 & 78 & $\mathrm{~F}$ & $-/-$ & Partial & Right & 3.7 & рT3a & NA & NA \\
\hline 5 & 69 & $M$ & $-/-$ & Partial & Left & 7.5 & $\mathrm{pT} 2 \mathrm{a}$ & 90 & AWD \\
\hline 6 & 83 & $\mathrm{~F}$ & $-/-$ & Radical & Right & 3.5 & pT1a & 49 & DAD \\
\hline 9 & 58 & $\mathrm{~F}$ & $-/-$ & Radical & Right & 5.5 & $\mathrm{pT} 1 \mathrm{~b}$ & NA & NA \\
\hline 10 & 76 & $\mathrm{~F}$ & $-/-$ & Partial & Right & 3.7 & pT1a & NA & NA \\
\hline
\end{tabular}

$F$ Female, $M$ Male, AWD Alive without recidive nor metastasis, NA Not available, $D A D$ Died of another disease (cardiovascular disease).

$(30 \%, 3 / 10)$ (Fig. 1E). All cases but one (90\%) contained one or more hypocellular area associated with edematous or more rarely fibromatous changes $(10 \%, 1 / 10)$ (Fig. 1F). Cells had homogeneous eosinophilic or oncocytic granular cytoplasms and exhibited uniformly round to oval nuclei, with perinuclear halo $(90 \%, 9 / 10)$ and sparse binucleations $(30 \%, 3 / 10)$ (Fig. 1G-H). Only three cases harbor focal dystrophic nuclei in areas closed to stroma changes, but all cases lacked typical nuclear features of CHRCC. Nucleoli were inconspicuous equivalent to WHO/ISUP 2. No mitotic activity was detected. Hemorrhage changes were frequent $(90 \%, 9 / 10)$, with vascularization exhibiting frequent focal sinusoid-like aspect $(70 \%, 7 / 10)$ and/or focal cystic change areas $(40 \%, 4 / 10)$ (Fig. 2A-B). Fibrohyaline changes were focally observed $(50 \%, 5 / 10)$ consisting in focal dense fibrosis bands like septa that delineate pseudo-nodules within the tumor (Fig. $2 C \pm D$ ). Interestingly, lymphocytic clusters were found in all but two cases $(80 \%, 8 / 10)$ (Fig. 2E-F). Two cases contained scattered small myoid-like nodules (Fig. 2G). Aggressive or adverse pathologic features, including vascular emboli or necrosis, were not seen. Two cases had a simple renal cyst in adjacent renal parenchyma.

\section{Immunohistochemical profile}

The 10 LOT exhibited diffuse positive CK7 (Fig. 3A) and negative CD117 (Fig. 3B) consistent with diagnostic criteria (Table 2). Expression of SDHB was retained (Fig. 3C). Phosphorylated and activated forms of 4EBP1 and S6K were overexpressed in 9 LOT (90\%) (H-score >100/300) (Fig. 3D-E) (Supplementary Fig. 2). Mean $\mathrm{H}$-score for 4EBP1-P and S6K-P was respectively 123.5 (range 5 to 200) and 135.3 (range 25 to 260). Expression of FOXI1 in tumoral cells was negative (Fig. $3 \mathrm{~F}, \mathrm{H}$ ) while positive internal control was available on intercalated cells of distal nephron either in corresponding available peritumoral section (Fig. 3F, G) or in other tumors on TMA (Table 2) (Supplementary Fig. 2).

\section{Genetic results}

The NGS of tumor DNA identified a genetic variant of interest in $80 \%(8 / 10)$ of cases (Table 3). Among them, $60 \%(6 / 10)$ were pathogenic activating MTOR mutations (c.4348 T > G p.(Tyr1450Asp), c.6644 C > T p.(Ser2215Phe), c.6644 C > A p.(Ser2215Tyr), c.7280 T>A p.(Leu2427Gln), c.7498 A > T p.(lle2500Phe) and c.7499T>A p.(lle2500Asn), previously reported in databases compiling somatic mutations in cancer. Those located on MTOR codons 2215 and 2500 are hotspot mutations. In tumor \#8, a MTOR inframe delins c.320_323delinsATT p.(Phe107_Ala108delinsTyrPhe) was found, considered as a variant of unknown significance. In one case (10\%), the genotyping revealed the presence of a truncating TSC1 pathogenic variant (c.2509_2512del
p.(Asn837Valfs*11)). The somatic status of mutations was confirmed in the 2 cases who benefited from both germline and tumor DNA NGS. However, variant allelic ratio were $<50 \%$, suggesting that each variant was somatic and absent from the germline DNA. No somatic variant of interest was identified in $20 \%$ $(2 / 10)$ of cases.

\section{DISCUSSION}

LOT has recently emerged from the spectrum of differential diagnosis between oncocytoma and eosinophilic variant of CHRCC $^{1,2,20,27}$. Based on two first reported cohorts since 2019 1,6 , LOT has been recently considered as a provisional entity by GUPS awaiting for more molecular characterization ${ }^{2}$.

In the current study, we describe a new cohort of 10 new cases of LOT and reveal that activation of mTOR pathway, due to MTOR activating or TSC1 inactivating mutations, plays a driver role in LOT oncogenesis. We also show that FOXI1 which is, as CD117, a lineage-specific marker of intercalated cells of distal nephron was negative in LOT. Moreover, we also provided additional clinicopathological data on this rare renal tumor and epidemiological data.

Diagnosis of LOT, as proposed in the original study performed by Trpkov et al., requires strict adequacy between immunohistochemical features that included diffuse CK7 and negative CD117 and "low grade" nuclear features closer to those of oncocytoma rather than $\mathrm{CHRCC}^{1}$. Using the same combination of morphological and immunohistochemical criteria, we have identified 10 new cases in a cohort of tumors previously diagnosed as CHRCC suggesting that incidence of LOT can be estimated at around 3.6\% of CHRCC. In two other cohorts, incidence of LOT was estimated to $3.7 \%$ of the oncocytic renal tumors ${ }^{3}$ and $4.18 \%$ (24/574) of renal oncocytoma ${ }^{4}$. However, in the cohort of 8 cases, Guo et al. have specified that diagnosis of one case (case 1) was based on immunoprofile profile $\mathrm{CK}^{+} / \mathrm{CD} 117^{-}$albeit nuclear features were more evocating of eosinophilic chromophobe with thickened plant cell-wall-like cytoplasmic borders and irregular or wrinkled nuclei ${ }^{6}$.

The meta-analysis of previous and current studies refines clinical data of LOT. LOT occurred more frequently in female (M:F ratio of $1: 1.3$ ) at mean age of 64.6 years. Indolent behavior of LOT is reinforced as no metastasis occurred during follow-up and only 3 tumors pT3a were reported ${ }^{1,3-6,28}$. However, if a metastasis were to occur, the presence of TSC/MTOR mutation could allow targeted therapy, as documented for metastatic eosinophilic solid and cystic RCC ${ }^{29}$.

In addition to the main morphological features characterizing LOT, we have also observed peculiar changes previously 

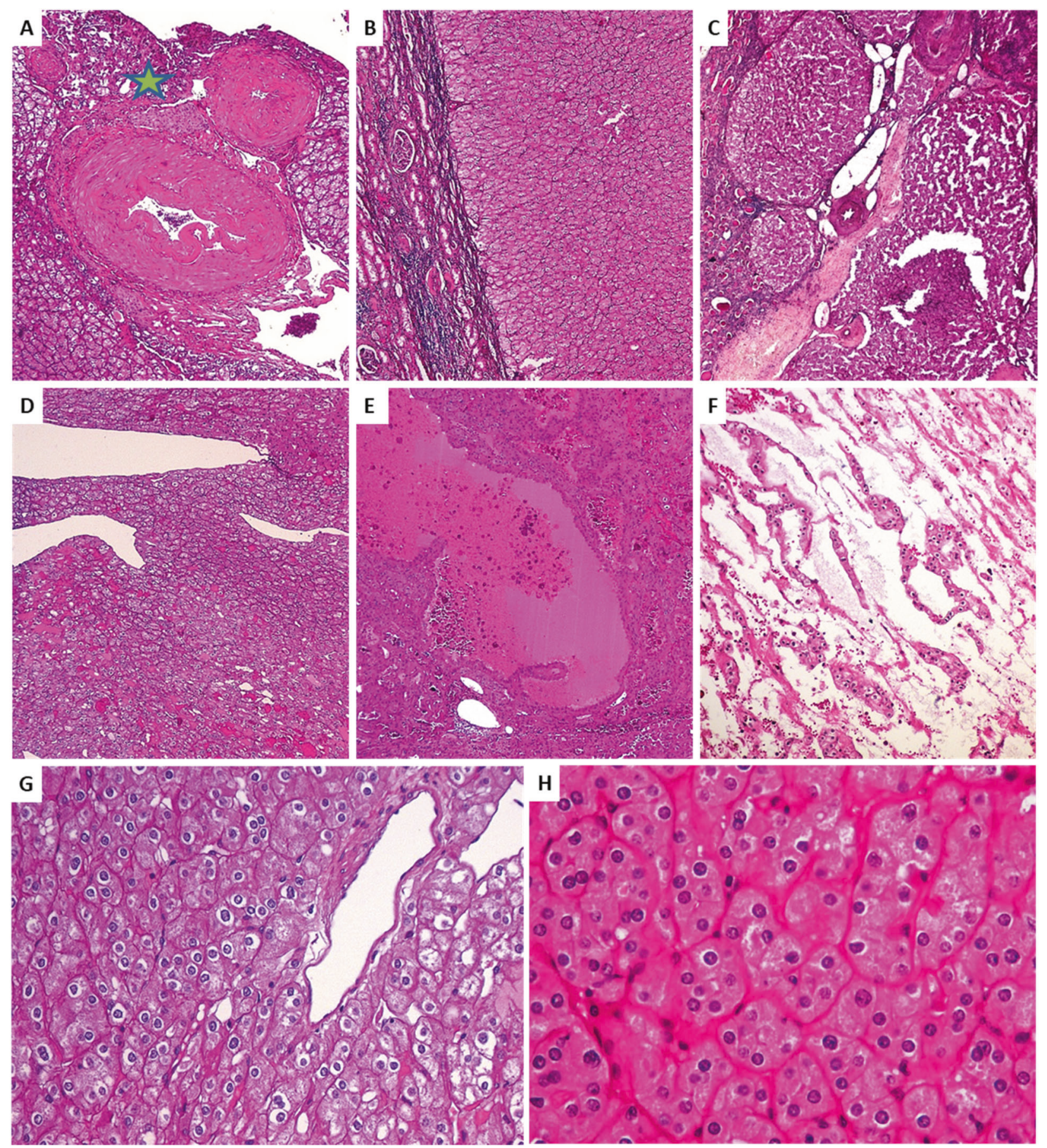

Fig. 1 Main microscopical features of LOT. All tumors are composed of eosinophilic/oncocytic cells (HES: A-H), one tumor was pT3a due to focal sinus invasion (A $\times 2.5$ from case 4, green star close to a nerve). All tumors were well-circumscribed (B $\times 5$ from case 5) or more rarely partially encapsulated with adjacent nodular extension (C $\times 2.5$ from case 6). Proliferation is organized in compact small nests containing frequent dilated vessels ( $\mathbf{D} \times 5$ from case 5 ) and may contain focal few microcystic area (E $\times 5$ from case 6$)$, or trabecular pattern (F from case 9 ). All cases but one contained one or more hypocellular area associated with edematous changes (F $\times 2.5$ from case 9). Tumoral cells had homogeneous eosinophilic or oncocytic granular cytoplasm and exhibited uniformly round to oval nuclei, with some perinuclear halo and sparse binucleations (G-H x 10-20 from case 5 and 7).

identified in stroma such as frequent edematous areas leading to hypocellular areas, as well as hemorrhage and frequent cluster of lymphocytes $(80 \% \text { of cases })^{1}$.

Our results obtained on FOXI1 expression provide further evidence to distinguish LOT from eosinophilic CHRCC and RO. Indeed, positivity of FOXI1 in CHRCC and RO that does originate from the intercalated cells have been extensively demonstrated in a recent large cohort and contrasted with its negativity in other RCC subtypes that does not originate from these cells ${ }^{18}$. Our results revealing a negative FOXI 1 staining in $100 \%$ of LOT cases are concordant with the negativity of CD117, another lineagespecific marker of intercalated cells of distal nephron shared by RO and $\mathrm{CHRCC}^{18}$. Hence, the combination of negative CD117/ negative FOXI1 allows to distinguish LOT from both RO and 

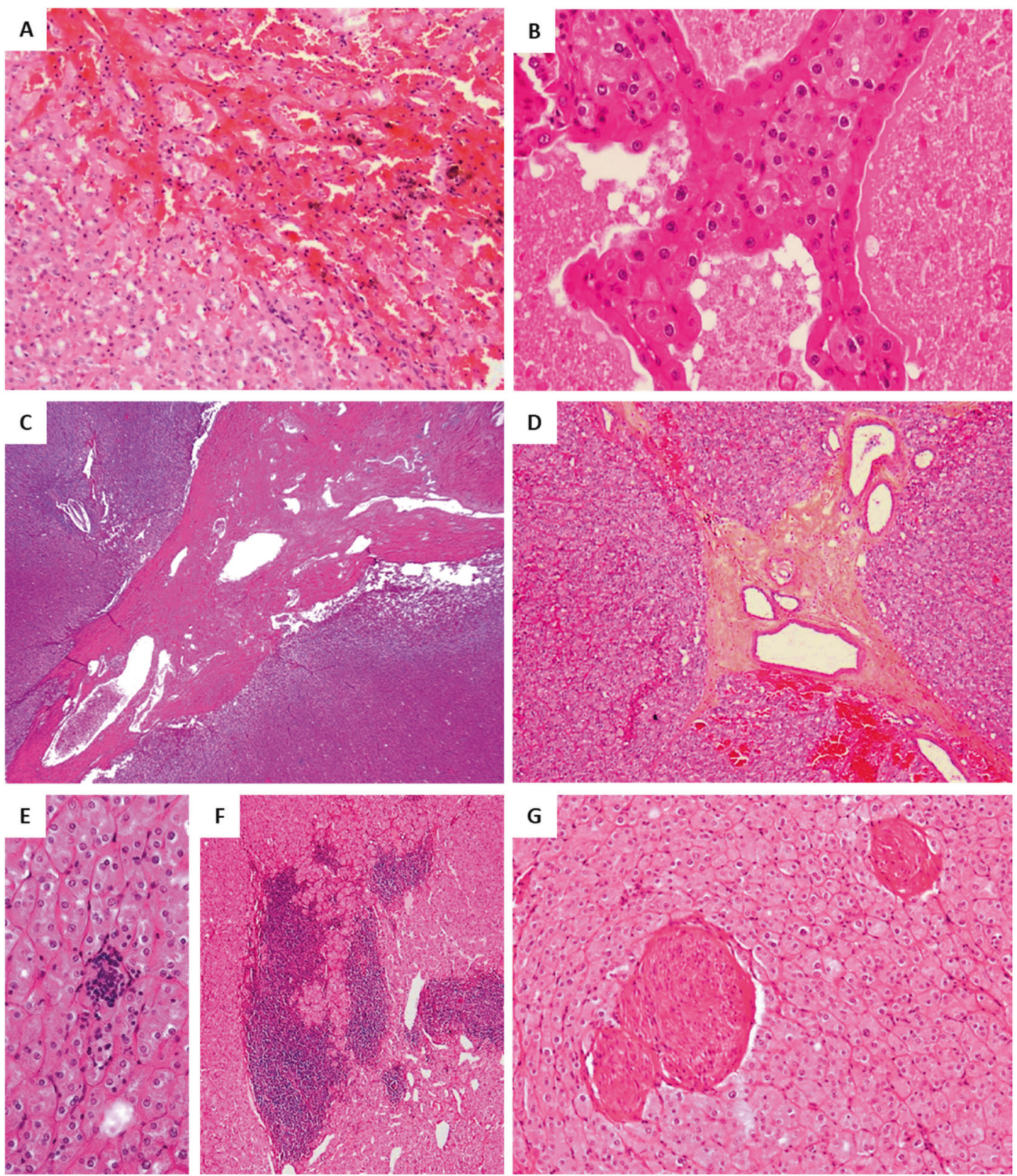

Fig. 2 Main features of stroma of LOT. Frequent hemorrhage changes given a sinusoid-like aspect (A $\times 10$ from case 3$)$ and/or focal cystic change areas (B $\times 10$ from case 3 ). Fibrohyaline changes were focally observed consisting in dense fibrosis bands like septa that delineate pseudo-nodules within the tumor (C $\times 10$ from case 3; D $\times 10$ from case 2). Small (E $\times 20$ from case 8) or large and multiple lymphocytic clusters were found in all but two cases (F X 20 from case 8) and focal small myoid like nodule in two cases (G X 20 from case 7) (A-G: HES).

eosinophilic CHRCC and also argues that LOT does not likely originate from the intercalated cells. Moreover, as previously reported $^{6}$, SDHB expression was retained.

Furthermore, our genetic results also argue to consider LOT as a distinct entity from CHRCC. Interestingly, we provide more evidence that molecular signature of LOT is assigned to mTOR pathway alterations. Variations in one mTOR pathway gene have been identified in $80 \%$ of our cases, mainly activating MTOR mutation (7/10). TSC1 inactivating mutation has been identified in one case. This genetic data differs from the main genetic alterations encountered in CHRCC, in which TP53 and PTEN are the two main genes mutated 24,30 .

In accordance with the activation of the mTORC1 pathway potentially induced by the genetic variations, we observed an overexpression of phosphorylated forms of 4EBP1 and S6K, two proteins downstream the mTOR pathway ${ }^{31}$. Of note that we have not identified any change in immunohistochemical or morphological features in the two cases of LOT without genetic variation compared to the 8 others. Among 9 cases exhibiting an overexpression ( $\mathrm{H}$-score $>100 / 300$ ) of the phosphorylated forms of 4EBP1 and S6K, 7 had a somatic mutation in MTOR or TSC genes. Conversely, $7 / 8$ of mutated cases demonstrated an overexpression of those 2 biomarkers. Compared to the results obtained in 9/10 LOT, H-score of 4EBP1-P and S6K-P in CHRCC included in the same TMA was negative or low (mean H-score respectively 42/300 and $50 / 300$ (data not shown)) reflecting, in our opinion, further argument for involvement of mTOR pathway in LOT. In CHRCC, overexpression of S6K-P has been poorly investigated. RoldanRomero et al. found an overexpression of S6K-P slightly more frequent in PTEN/FLCN-mutated tumors (8/16 cases, 50\%) compared with tumors without mutations in mTOR pathway related genes $(13 / 41,32 \%)^{32}$. 

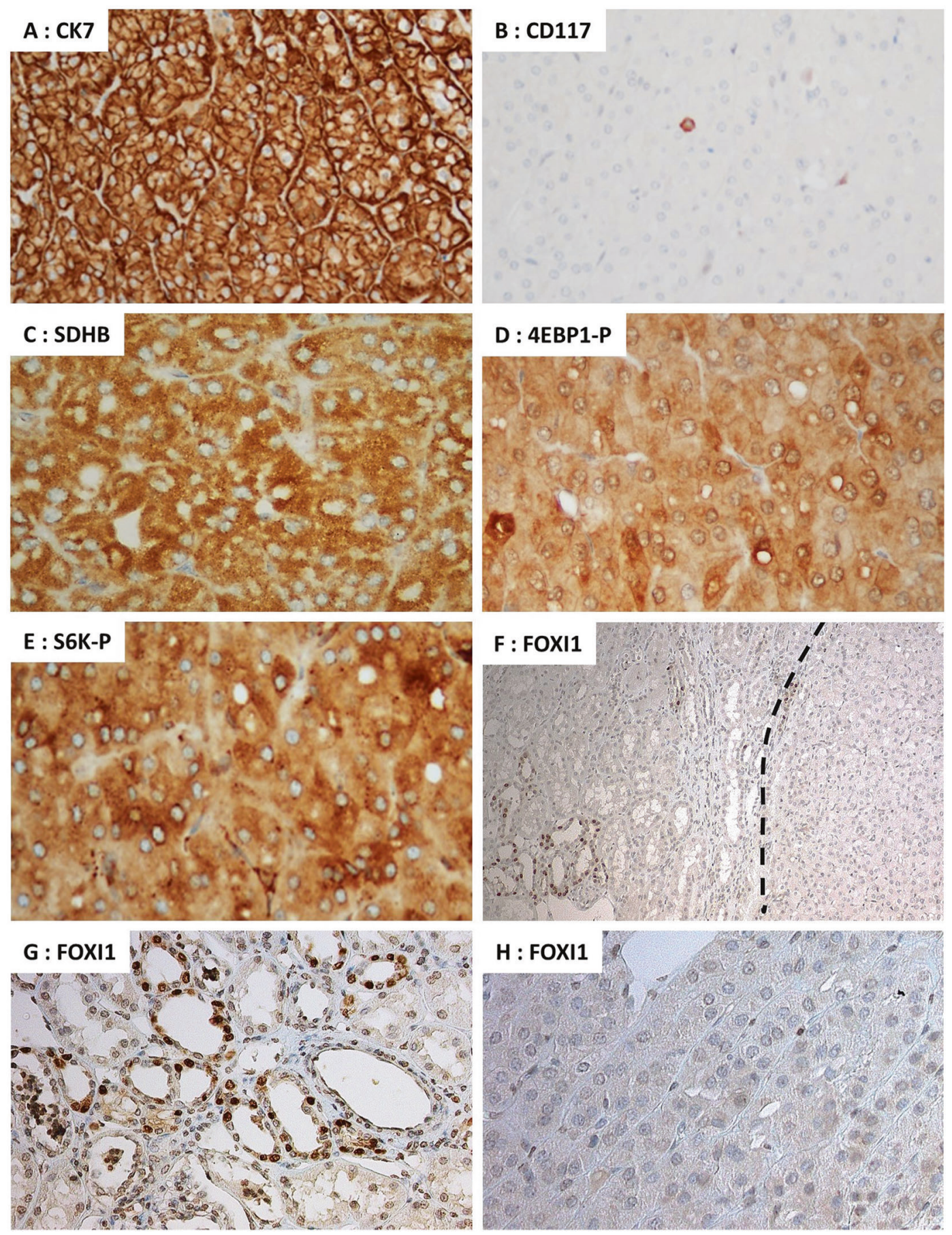

Fig. 3 Immunohistochemical profile of LOT with CK7, CD117, SDHB, 4EBP1-P, S6K-P, and FOXI1 antibodies. All tumors harbor diffuse expression of CK7 $(\mathbf{A} \times 10)$ and negative expression of CD117 with are few positive mastocytes (B $\times 10)$, a retained cytoplasmic expression of SDHB (C x 10). Phosphorylated and activated forms of 4EBP1 (D) and S6K (E) were overexpressed in 9 LOT (D-E x 10). Expression of FOXI1 was positive in internal control on intercalated cells of distal nephron of peritumoral section ( $\mathbf{F}$ and $\mathbf{G} \times \mathbf{1 0}$ ) whereas tumoral cells were negative or $<1 \%$ of cells in all LOT ( $\mathbf{F}$ and $\mathbf{H} \times 10)$.

In other eosinophilic renal tumors associated with mutation in MTOR or TSC1/2 genes such as eosinophilic vacuolated tumor and eosinophilic solid and cystic RCC, overexpression of both phospho-4EBP1 and phospho-S6K has also been identified ${ }^{33,34}$.

As we identified one case of LOT with somatic TSC1 mutation, we reviewed features of 'eosinophilic CHRCC' described in the two large series of RCC occurring in TSC patients published in 2014 with the objective to identify some eventual similarities with LOT $^{35,36}$. In one cohort, Guo et al. have described a subset of tumor with CHRCC-like morphology in which 31 of 34 tumors "resembled the eosinophilic variant of chromophobe". A diffusely positive CK7 was observed in 6/7 cases whereas CD117 "reactivity was only noted in 1 of 6 ". In each case, few perinuclear halos, binucleation and scattered foci of hyperchromatic and irregular nuclei were seen associated with large areas with more monomorphic nuclear features similar to oncocytoma ${ }^{35}$. In the second series of RCC associated with TSC, Yang et al. described a group of 15 eosinophilic tumors morphologically similar to 
Table 2. Immunohistochemical profile of LOT.

\begin{tabular}{|c|c|c|c|c|c|c|c|}
\hline Case & Diffuse CK7 + & Negative CD117 & Retained SDHB & $\begin{array}{l}\text { FOXI1 } \\
\text { TC }\end{array}$ & $\begin{array}{l}\text { FOXI1 } \\
\text { Positive control IC }\end{array}$ & $\begin{array}{l}\text { 4EBP1-P } \\
\text { H-score* }\end{array}$ & $\begin{array}{l}\text { S6K-P } \\
\text { H-score* }\end{array}$ \\
\hline 1 & Yes & Yes & Yes & - & Yes & + & + \\
\hline 2 & Yes & Yes & Yes & - & Yes & ++ & + \\
\hline 3 & Yes & Yes & Yes & - & $N A^{*}$ & + & + \\
\hline 4 & Yes & Yes & Yes & - & Yes & + & + \\
\hline 5 & Yes & Yes & Yes & - & Yes & + & + \\
\hline 8 & Yes & Yes & Yes & - & $N A^{*}$ & + & + \\
\hline 9 & Yes & Yes & Yes & - & Yes & - & - \\
\hline 10 & Yes & Yes & Yes & - & Yes & + & + \\
\hline
\end{tabular}

FOXI1: (-): Negative < $1 \%$, focal 1 to $25 \%$, moderate 26 to $50 \%$, diffuse 51 to $100 \%$

TC Tumoral Cells, IC Intercalated cells of distal nephron in peritumoral kidney, NA* Not available for this sample but positive on other samples on the same TMA

H-score*: 4EBP1-P, S6K-P: (-): negative or low expression <100/300), $(+/++)$ positive and overexpressed; $(+)$ : $\geq 100<200 / 300 ;(++): \geq 200 / 300$.

Table 3. Results of the genetic analysis in the cohort using a panel of 26 genes by NGS.

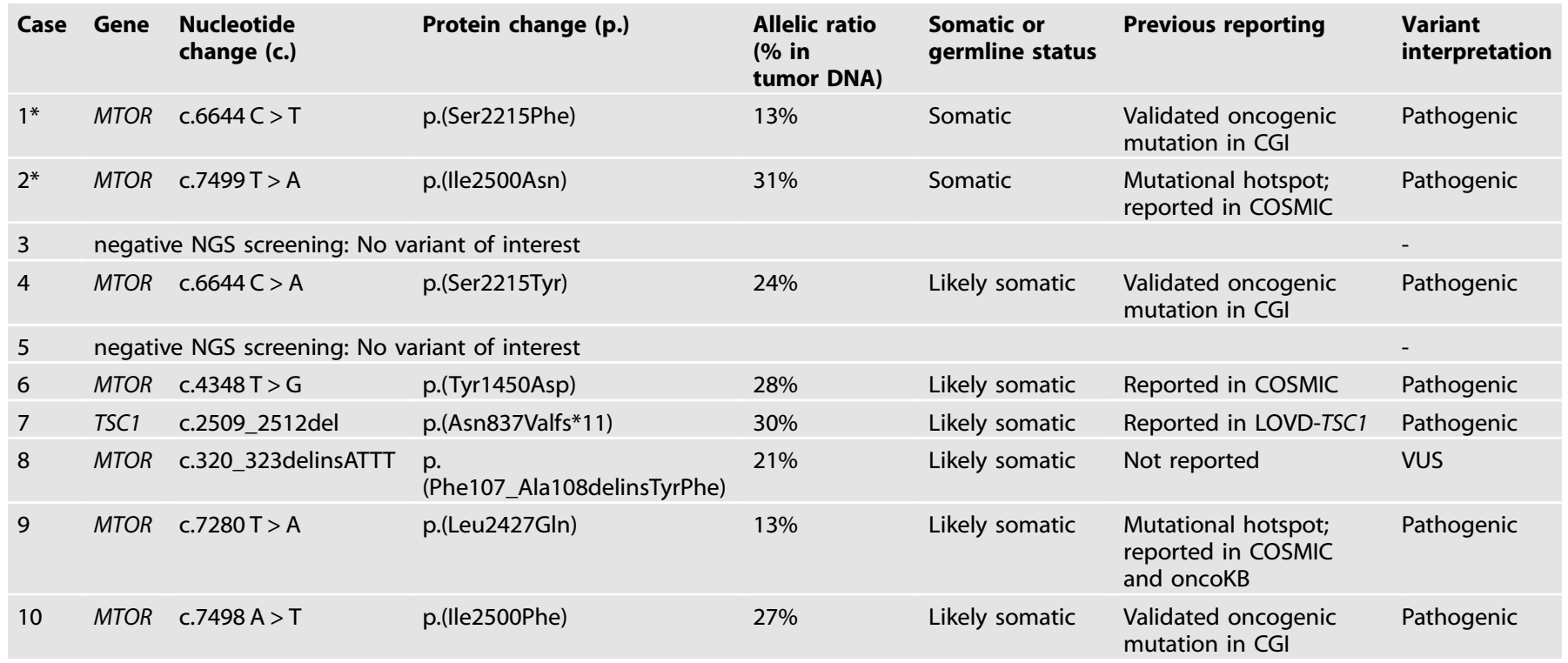

*Both tumor and germline DNAs were analyzed, Likely somatic: no germline analysis but allelic ratio $<50 \%$, VUS variant of unknown significance, CGI Cancer Genome Interpreter, COSMIC Catalogue Of Somatic Mutations In Cancer, oncoKB Precision Oncology Knowledge Base

hybrid oncocytic/chromophobe tumors (HOCT) but none of them displayed diffuse CK7 positivity and CD117 negativity observed in $\mathrm{LOT}^{36}$. In contrast with other recently described eosinophilic tumors such as 'eosinophilic solid and cystic renal cell carcinomas' and 'eosinophilic vacuolated renal tumor/high grade oncocytic tumors' ${ }^{2}$ in which tuberous sclerosis complex setting has been reported, no hereditary syndrome is associated to LOT in our cohort. However, 4 cases of multiple LOT have been described very recently and for the first time in the setting of $\mathrm{TSC}^{4,5}$. In Lerma et al., 2 patients with multiple LOT (one with $3 \mathrm{LOT}<0.5 \mathrm{~cm}$ and one with 9 bilateral from 0.3 to $5 \mathrm{~cm}$ ) and other related TSCtumors have been identified to carry TSC1 pathogenic mutation (one [c.2074 C > (p.Arg692*)], and one heterozygous [c.395_406del ins CA.Gly132Alafs*2)] $]^{5}$. Two other patients with multiple LOT have been also reported in the cohort of 29 LOT from the Mayo Clinic ${ }^{4}$. Of note, Gupta et al. have identified 3 more TSC patients with 9 indolent oncocytic tumors harboring diffuse $C K 7^{+}$low-absent
CD117 (range from 0.5 to $6 \mathrm{~cm}$ ) but "lacking irregular nuclei of CHRCC" and "edematous stroma with loosely arranged cells" of LOT that they have classified as TSC-associated oncocytic tumor ${ }^{37}$. Further studies will be necessary to determine the incidence of TSC1/2 somatic and germline mutations in LOT.

Finally, our results highlight potential overlap between LOT and recent other cases of renal tumors initially diagnosed as eosinophilic CHRCC harboring either MTOR or TSC1/2 mutation. For 32 cases identified in 6 recent molecular studies ${ }^{18,19,32,38-40}$, we have recapitulated available morphological, immunohistochemical, mutational, and chromosomal copy number variation data, in order to compare them with LOT features (Supplementary Table 3). Some of those cases have been discussed in the two recent GUPS review on renal tumors ${ }^{2,20}$. Similarly to LOT, none of the 32 cases harbored driver RCC-associated mutations except in MTOR or TSC1/2 genes. We have identified a total of six cases associating FOXI1 low or null expression and either TSC1 mutation 
(1 case) or MTOR mutation (5 cases) ${ }^{18,19}$. Eosinophilic CHRCC with both negative FOXI1 and MTOR mutation include the two first cases reported by Skala et al. ${ }^{18}$ and four cases from TCGA (KN8437; KM-8441; KM-8639; MH-A857) reported by Tong et al. ${ }^{19}$. At least five of them exhibited a diploid pattern. Unfortunately, we have not found data regarding CK7/CD117 status for those cases, but morphological features shared those of LOT criteria. Tong et al. proposed the revised diagnosis of eosinophilic chromophobe-like renal tumor for their four tumors and Skala et al. pointed out that their two cases mimicked eosinophilic $\mathrm{CHRCC}^{18,19}$. Finally, their lack of CK7/CD117 status could not determine whether they are LOT or not.

Other remarkable cases concern at least five eosinophilic tumors (among 12 tumors that belong to the group named "group 2 " by Tjota et al. $\left.{ }^{39}\right)$. Those five tumors (2-I, 2-Ila, 2-IIB, 2-V and 2-VII) developed in 4 patients share with LOT, positivity of CK7 (at least described as greater than $50 \%$ ), and negativity of CD117 and were morphologically solid, composed by cells with abundant eosinophilic cytoplasm, round nuclei, inconspicuous nucleoli, occasional binucleation, and clear perinuclear halo ${ }^{39}$. However, one patient had multiple tumors and a larger tumor classified pT2b. All cases harbored MTOR (one case), TSC2 (one case) or TSC1 ( 2 cases) mutations. Moreover, none of them have classical profile of multiple chromosomal losses of CHRCC. However, one patient had multiple tumors and larger tumors classified pT2b. Considering these criteria, all these 5 cases could be reclassified as LOT.

In conclusion, our study provides evidence to consider mTOR pathway genes as driver genes of tumorigenesis of LOT, expanding the spectrum of emergent eosinophilic/oncocytic renal tumors associated with alterations in mTOR pathway. In our study, these mTOR pathway genetic alterations are mainly represented by MTOR activating mutations (60\%) but also included one TSC1 truncating variant (10\%). In addition, the current report shows the absence of FOXI1 expression in LOT providing another evidence to distinguish them from eosinophilic variant of CHRCC that originates from distal nephron. Our results allow improving the diagnosis of LOT which combine homogeneous diffuse CK7 positivity, CD117, and FOXI1 negativity. This specific immunoprofile is associated with MTOR or TSC1/2 somatic mutations in a large majority of cases. Finally, our results argue to consider LOT as a distinct subtype of oncocytic/eosinophilic tumors, sharing sporadic presentation and favorable clinical outcome. Consequently, the accurate diagnosis of LOT is essential for the patient's management that would be altered in case of metastatic evolution. Indeed, if a metastasis were to occur, the presence of TSC/MTOR mutation could allow targeted therapy.

\section{DATA AVAILABILITY}

All data generated or analyzed during this study are included in this published article [and its supplementary information files].

\section{REFERENCES}

1. Trpkov, K. et al. Low-grade oncocytic tumour of kidney (CD117-negative, cytokeratin 7-positive): a distinct entity? Histopathology 75, 174-184 (2019).

2. Trpkov, K. et al. Novel, emerging and provisional renal entities: the Genitourinary Pathology Society (GUPS) update on renal neoplasia. Mod. Pathol. 34, 1167-1184 (2021).

3. Akgul, M., Al-Obaidy, K. I., Cheng, L., \& Idrees, M. T. Low-grade oncocytic tumour expands the spectrum of renal oncocytic tumours and deserves separate classification: a review of 23 cases from a single tertiary institute. J. Clin. Pathol. https://doi.org/10.1136/jclinpath-2021-207478 (2021).

4. Kravtsov, O. et al. Low-grade oncocytic tumor of kidney (CK7-positive, CD117negative): incidence in a single institutional experience with clinicopathological and molecular characteristics. Hum. Pathol. 114, 9-18 (2021).

5. Lerma, L. A., Schade, G. R., \& Tretiakova, M. S. Co-existence of ESC-RCC, EVT, and LOT as synchronous and metachronous tumors in six patients with multifocal neoplasia, but without clinical features of tuberous sclerosis complex. Hum. Pathol. 116, 1-11 (2021).
6. Guo, Q. et al. Characterization of a distinct low-grade oncocytic renal tumor (CD117-negative and cytokeratin 7-positive) based on a tertiary oncology center experience: the new evidence from China. Virchows Arch 478, 449-458 (2021).

7. Kryvenko, O. N., Jorda, M., Argani, P. \& Epstein, J. I. Diagnostic approach to eosinophilic renal neoplasms. Arch Pathol. Lab. Med. 138, 1531-1541 (2014).

8. Williamson, S. R. et al. Diagnostic criteria for oncocytic renal neoplasms: a survey of urologic pathologists. Hum. Pathol. 63, 149-156 (2017).

9. Wobker, S. E. \& Williamson, S. R. Modern pathologic diagnosis of renal oncocytoma. J. Kidney Cancer VHL 4, 1-12 (2017).

10. Zhou, J., Yang, X., Zhou, L., Zhang, P. \& Wang, C. Combined immunohistochemistry for the "Three 7 » Markers (CK7, CD117, and Claudin-7) is useful in the diagnosis of chromophobe renal cell carcinoma and for the exclusion of mimics: diagnostic experience from a single institution. Dis. Markers 2019, 4708154 (2019).

11. Zhao, W. et al. DOG1, cyclin D1, CK7, CD117 and vimentin are useful immunohistochemical markers in distinguishing chromophobe renal cell carcinoma from clear cell renal cell carcinoma and renal oncocytoma. Pathol. Res. Pract. 211, 303-307 (2015).

12. $\mathrm{Ng}, \mathrm{K}$. L. et al. A systematic review and meta-analysis of immunohistochemical biomarkers that differentiate chromophobe renal cell carcinoma from renal oncocytoma. J. Clin. Pathol. 69, 661-671 (2016).

13. Huo, L. et al. C-kit expression in renal oncocytomas and chromophobe renal cell carcinomas. Hum. Pathol. 36, 262-268 (2005).

14. Din, N. U., Fatima, S. \& Ahmad, Z. Chromophobe renal cell carcinoma: a morphologic and immunohistochemical study of 45 cases. Ann. Diagn. Pathol. 17, 508-513 (2013).

15. Yamazaki, K. et al. Overexpression of KIT in chromophobe renal cell carcinoma. Oncogene 22, 847-852 (2003).

16. Zimpfer, A. et al. C-kit overexpression is not associated with KIT gene mutations in chromophobe renal cell carcinoma or renal oncocytoma. Pathol. Res. Pract. 210, 521-525 (2014).

17. Lindgren, D. et al. Cell-type-specific gene programs of the normal human nephron define kidney cancer subtypes. Cell Rep. 20, 1476-1489 (2017).

18. Skala, S. L. et al. Next-generation RNA sequencing-based biomarker characterization of chromophobe renal cell carcinoma and related oncocytic neoplasms. Eur. Urol. 78, 63-74 (2020).

19. Tong, K. \& Hu, Z. FOXI1 expression in chromophobe renal cell carcinoma and renal oncocytoma: a study of The Cancer Genome Atlas transcriptome-based outlier mining and immunohistochemistry. Virchows Arch 478, 647-658 (2020).

20. Trpkov, K. et al. New developments in existing WHO entities and evolving molecular concepts: the Genitourinary Pathology Society (GUPS) update on renal neoplasia. Mod. Pathol. 34, 1392-1424 (2021).

21. Ohashi, R. et al. Classic chromophobe renal cell carcinoma incur a larger number of chromosomal losses than seen in the eosinophilic subtype. Cancers 11, 1492-1506 (2019).

22. Thoenes, W. et al. Chromophobe cell renal carcinoma and its variants-a report on 32 cases. J. Pathol. 155, 277-287 (1988).

23. Amin, M. B. et al. Chromophobe renal cell carcinoma: histomorphologic characteristics and evaluation of conventional pathologic prognostic parameters in 145 cases. Am. J. Surg. Pathol. 32, 1822-1834 (2008).

24. Davis, C. F. et al. The somatic genomic landscape of chromophobe renal cell carcinoma. Cancer Cell 26, 319-330 (2014).

25. Alaghehbandan, R. et al. Comprehensive review of numerical chromosomal aberrations in chromophobe renal cell carcinoma including its variant morphologies. Adv. Anat. Pathol. 28, 8-20 (2021).

26. Morini, A. et al. Immunohistochemical evaluation of the mTOR pathway of genetically characterized chromophobe renal cell carcinomas: a pilot study of 20 cases. abstracts from uscap 2020: genitourinary pathology (860-1046). Mod. Pathol. 33, 1002-1063 (2020).

27. Siadat, F. \& Trpkov, K. ESC, ALK, HOT and LOT: three letter acronyms of emerging renal entities knocking on the door of the WHO classification. Cancers 12, 168-184 (2020).

28. de Andrade Melo, C. G. R., Xavier, M. V. N., Pimenta, I. S. \& Athanazio, D. A. Lowgrade oncocytic tumour of kidney (CD117-negative, cytokeratin 7-positive). Surg. Exp. Pathol. 3, 22 (2020).

29. Palsgrove, D. et al. Eosinophilic Solid and Cystic (ESC) renal cell carcinomas harbor TSC mutations: molecular analysis supports an expanding clinicopathologic spectrum. Am. J. Surg. Pathol. 42, 1166-1181 (2018).

30. Ricketts, C. J. et al. The Cancer Genome Atlas comprehensive molecular characterization of renal cell carcinoma. Cell Rep. 23, 313-326 (2018).

31. Saxton, R. A. \& Sabatini, D. M. mTOR signaling in growth, metabolism, and disease. Cell 168, 960-976 (2017).

32. Roldan-Romero, J. M. et al. Molecular characterization of chromophobe renal cell carcinoma reveals mTOR pathway alterations in patients with poor outcome. Mod. Pathol. 33, 2580-2590 (2020). 
33. Chen, Y.-B. et al. Somatic mutations of TSC2 or MTOR characterize a morphologically distinct subset of sporadic renal cell carcinoma with eosinophilic and vacuolated cytoplasm. Am. J. Surg. Pathol. 43, 121-131 (2019).

34. Mehra, R. et al. Somatic Bi-allelic loss of TSC genes in eosinophilic solid and cystic renal cell carcinoma. Eur. Urol. 74, 483-486 (2018).

35. Guo, J. et al. Tuberous sclerosis-associated renal cell carcinoma: a clinicopathologic study of 57 separate carcinomas in 18 patients. Am. J. Surg. Pathol. 38, 1457-1467 (2014).

36. Yang, P. et al. Renal cell carcinoma in tuberous sclerosis complex. Am. J. Surg. Pathol. 38, 895-909 (2014).

37. Gupta, S. et al. Renal neoplasia in tuberous sclerosis: a study of 41 patients. Mayo Clin. Proc. 96, 1470-1489 (2021).

38. TCGA-KICH: https://portal.gdc.cancer.gov/projects/TCGA-KICH

39. Tjota, M. et al. Eosinophilic renal cell tumors with a TSC and MTOR gene mutations are morphologically and immunohistochemically heterogenous: clinicopathologic and molecular study. Am. J. Surg. Pathol. 44, 943-954 (2020).

40. Tjota, M. Y., Wanjari, P., Segal, J. \& Antic, T. TSC/MTOR mutated eosinophilic renal tumors are a distinct entity that are CK7+/CK20-/vimentin-: a validation study. Hum. Pathol. 115, 84-95 (2020).

\section{ACKNOWLEDGEMENTS}

We wanted to thank Marine Largeau from the biological Resources Center and Tumor Bank Platform (BB-0033-00063) of Georges Pompidou European Hospital, Gisele Legall from the biological Resources Center and Tumor Bank Platform of Necker Enfants Malades Hospital, Sophie Hajosi and technicians from the Department of Pathology of Georges Pompidou European Hospital for technical assistance for TMA.

\section{FUNDING INFORMATION}

Immunohistochemistry of mTOR pathway and Tom Drossart was partly funded by Fondation ARC (PGA1 RF20190208466).

\section{AUTHOR CONTRIBUTIONS}

V.V.E. performed study concept and design. A.P.G.R., A.M.E., A.M.O., and M.O.T. provided acquisition of clinical data. A.M.O., J.F., M.S., V.V.A., and V.V.E. provided acquisition of pathological and immunohistochemical data. A.P.G.R., N.B., and T.D. provided acquisition of genetic analysis. C.B. and J.F. provided technical and material support. A.M.O., N.B., and V.V.E. performed development of methodology, writing, review, and revision of the paper. A.M.O., N.B., T.D., and V.V.E. provided analysis, interpretation of data, and statistical analysis. All authors read and approved the final paper.

\section{ETHICS APPROVAL AND CONSENT TO PARTICIPATE}

Our study was approved by the French national ethics committee (Comité de Protection des Personnes Number 19.05.27.61541; national identification 2018-A03147-48).

\section{COMPETING INTERESTS}

The authors declare no competing interests.

\section{ADDITIONAL INFORMATION}

Supplementary information The online version contains supplementary material available at https://doi.org/10.1038/s41379-021-00906-7.

Correspondence and requests for materials should be addressed to Virginie Verkarre.

Reprints and permission information is available at http://www.nature.com/ reprints

Publisher's note Springer Nature remains neutral with regard to jurisdictional claims in published maps and institutional affiliations. 